\title{
The Analysis of Safety and Effectiveness Using Allograft in the Treatment of Spinal Tuberculosis-A Multicenter Retrospective Study
}

\author{
Sen YANG, Hongwei LU, Fei LUO, Zehua ZHANG, Wenjie WU \\ Third Military Medical University, Southwest Hospital, Department of Orthopaedics, Chongqing, China \\ *Sen Yang and Hongwei Lu contributed equally to this work and should both be considered first authors. Other authors contributed equally to this work. \\ This study has been presented as a poster at the $33^{\text {rd }}$ Annual Meeting of the North American Spine Society between 26 and 29 September 2018 at Los \\ Angeles, California, USA
}

Corresponding author: Wenjie WU bisheng320@126.com

\section{ABSTRACT}

AIM: To evaluate the clinical efficacy and fusion characteristics of allografts in spinal tuberculosis (TB).

MATERIAL and METHODS: The research reviewed 1196 patients with spinal tuberculosis who received treatment at six hospitals from January 2000 to January 2016. A total of 623 patients who had spinal tuberculosis were included in the study. All patients underwent debridement, decompression, allograft bone grafting, and instrumentation. Postoperative treatment consisted of a combination of anti-TB drug treatment for 18 months and brace fixed braking for 3-9 months. Clinical outcome, laboratory indexes, and radiological results were analysed.

RESULTS: The average follow-up time was 34.1 months (12-60 months). Pain was relieved postoperatively in all cases, and $87.8 \%$ of patients were painless at the final follow-up. The erythrocyte sedimentation rate (ESR) significantly decreased and returned to normal at the final follow-up. The fusion rate of allografts was $30.2 \%$ and $98.4 \%$ at the 9 - and 12 -month follow-ups, respectively. At the final follow-up, the fusion rate was $100 \%$.

CONCLUSION: The application of allografts in the surgical management of spinal tuberculosis is safe and effective. Allografts can replace autografts in surgeries for spinal tuberculosis.

KEYWORDS: Allograft, Fusion, Spinal tuberculosis

ABBREVIATIONS: ESR: Erythrocyte sedimentation rate, VAS: Visual analogue pain scale, ASIA: American Spinal Injury Association,

CT: Computed tomography

\section{INTRODUCTION}

$P^{j}$ pinal tuberculosis, the most common type of bone and joint tuberculosis, accounts for $50 \%$ of all cases of skeletal tuberculosis, which frequently causes neurologic deficits, kyphotic deformity, or even paraplegia (18). In recent years, anti-tuberculosis drugs have become more standardized in the treatment of spinal tuberculosis and have achieved good therapeutic effects. However, complications, such as spinal instability, kyphosis and neurological dysfunction, often occur in follow-up and usually require surgical treatment (29). However, after debridement, the choice of surgical treatment used to place an allograft or autograft into the bone defect area remains controversial $(13,40)$.
Sen YANG (1): 0000-0002-0755-4968

Hongwei LU (1) : 0000-0002-3294-4669

Fei LUO (D) : 0000-0002-6368-6674
Zehua ZHANG (1D : 0000-0003-0662-4490

Wenjie WU (1) : 0000-0002-8014-5335 
While an autograft remains the "gold standard", the limitation with autografts is supply, especially when there are large bone defects after debridement. Furthermore, operative time and blood loss increase due to bone harvesting, donor site complications often occur, and postoperative pain tends to prolong the patient's time spent bedridden and impact the patient's rehabilitation. Allografts are relatively abundant, and their supporting strength is ideal. However, their doubted their clinical efficacy (31). Some authors have shown that placement of allografts in the setting of spinal infections can be performed safely $(1,23,35)$; however, the sample size is small and lacks sufficient persuasion. Furthermore, in the current literature, there have been fewer multicentre studies on the use of cages with allografts in spinal tuberculosis. Therefore, we reviewed patients with spinal tuberculosis from six hospitals and observed the efficacy and safety of allografts. These findings also provide a reference for the selection of bone grafts in spinal tuberculosis fusion.

\section{MATERIAL and METHODS}

This research was approved by the Ethics Committee of the First Affiliated Hospital of the Third Military Medical University, People's Liberation Army (Protocol number: KY201301). A total of 1196 consecutive patients with spinal infections from six hospitals between 2000 and 2016 were reviewed for this research. Inclusion criteria: diagnosis of spinal tuberculosis (based on clinical features, laboratory indices, pathological analysis, and radiology results), received surgical treatment using an allograft, had no complicated active pulmonary tuberculosis or tumours, patient compliance was good, and postoperative follow-up was at least 12 months. In total, 623 patients were analysed in the study, including 329 men and 294 women, with an average age of $37.1 \pm 16.8$ years (range 2-80 years). Apart from 17 patients with skipped lesions, 23 cases had cervical involvement, 230 cases involved the thoracic spine, 72 cases involved the thoracolumbar spine (T11-L2) and 281 cases involved the lumbar spine. The pathogenic vertebral levels consisted of multiple lesions in 44 cases, with four contiguous vertebrae in 66 cases, three contiguous vertebrae in 97 cases, two contiguous vertebrae in 346 cases and one vertebrae in 70 cases.

The surgical protocols included the anterior approach, the posterior approach, and the combined anterior-posterior approach. The surgical procedures were performed according to standard surgical approaches $(6,10,16,17,32)$. Debridement, decompression, and placement of an allogenic osteoinduction is relatively weak, and some researchers have

iliac crest, fibular graft, polyether-ether-ketone (PEEK) cage with allograft, or bone intervertebral fusion cage made by allogenic cortical bone (Figure 1) was performed according to the extent of the lesion area. Suitable instrumentation was implanted and the incision was closed. In total, 171 patients underwent the anterior approach, while the rest were treated with either the combined approach (135 patients) or the posterior approach (317 patients). Surgeons at each hospital made decisions about the selection of surgical methods and instruments, and the surgeries were performed by the same team at each respective hospital.

Treatment of tuberculosis is the primary goal, so appropriate anti-tuberculosis drugs were administered in all patients after consult recommendations, which usually included at least 2 weeks of orally administered HREZ (rifampicin, $15 \mathrm{mg} /$ $\mathrm{kg}$, maximum $600 \mathrm{mg} /$ day; isoniazid, $6 \mathrm{mg} / \mathrm{kg}$, maximum 300 mg/day; ethambutol, 25 mg/kg, maximum 750 mg/day; and pyrazinamide $25 \mathrm{mg} / \mathrm{kg}$, maximum $750 \mathrm{mg} /$ day) before surgery and continued until 18 months after surgery. Among these patients, 36 had drug-resistant spinal tuberculosis, and their regimen was adjusted according to the results of drug sensitivity testing (4).

The erythrocyte sedimentation rate (ESR) monitored infections, visual analogue pain scale (VAS) and American Spinal Injury Association (ASIA) assessed clinical efficacy and neurologic function, and computed tomography (CT) assessed bone fusion (according to the modified radiologic criteria) (21).

\section{Statistical Analysis}

All analyses were performed using SPSS 19.0 (SPSS Inc, Chicago, IL, USA). VAS scores were compared with ANOVA, followed by Dunnett's T3 test or LSD t test to compare preoperative, postoperative and last follow-up values. $P$ values of less than 0.05 were considered statistically significant.

\section{RESULTS}

Table I: Demographic Data of Patients

\begin{tabular}{lc}
\hline Characteristic & Total $(\mathbf{n}=623)$ \\
\hline \multicolumn{1}{l}{ Sex } & \\
\hline Male & 329 \\
\hline Female & 294 \\
\hline Age (years) & $37.1 \pm 16.8(2-80)$ \\
\hline Follow-up (months) & $34.12 \pm 14.05$ \\
\hline
\end{tabular}
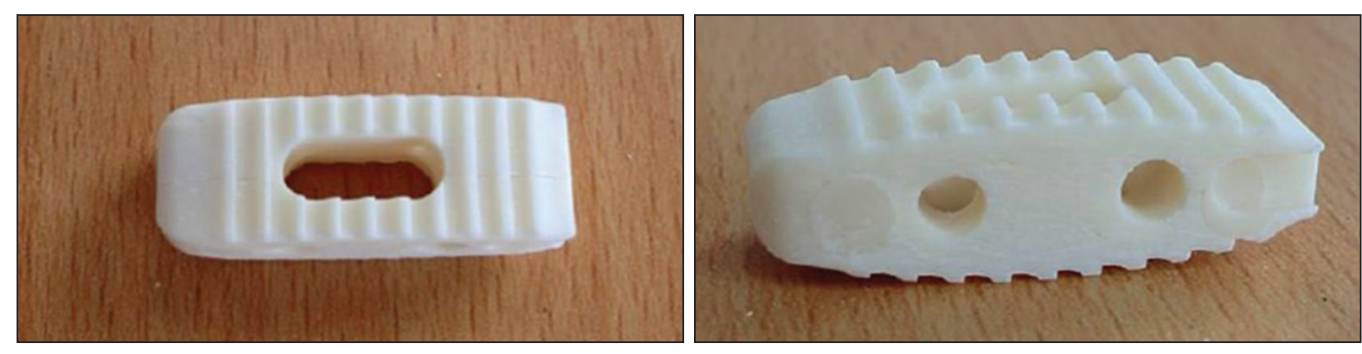

Figure 1: The intervertebral fusion cage made by allogenic cortical bone. 


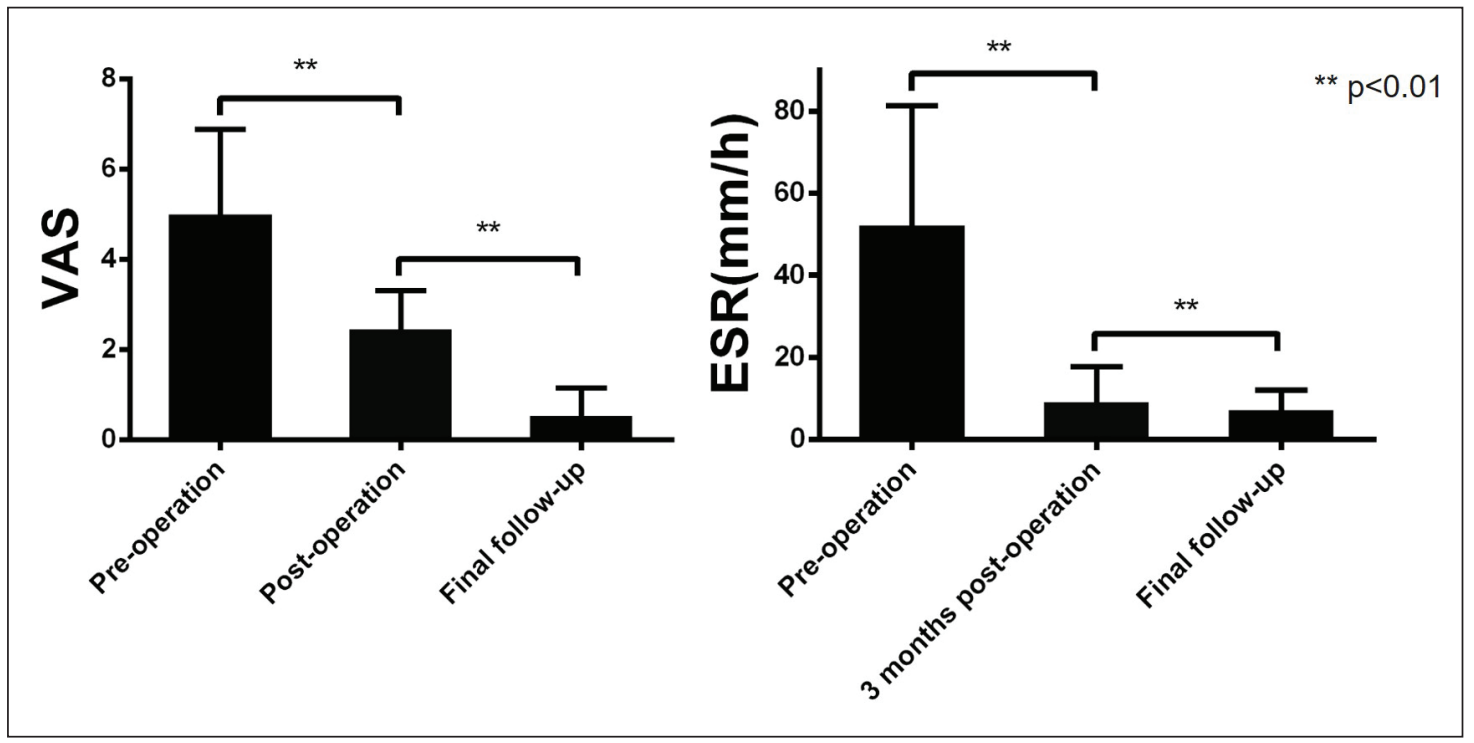

Figure 2: The graph shows the change of the visual analogue pain scale (VAS) score and erythrocyte sedimentation rate (ESR) preoperatively, postoperatively and at final follow-up.

Table II: Neurologic Recovery according to ASIA

\begin{tabular}{|c|c|c|c|c|c|c|c|c|c|c|c|c|c|c|c|}
\hline \multirow{2}{*}{ ASIA grade } & \multicolumn{5}{|c|}{ Preoperative } & \multicolumn{5}{|c|}{ Postoperative } & \multicolumn{5}{|c|}{ Final follow-up } \\
\hline & A & B & C & D & E & A & B & C & D & $\mathbf{E}$ & A & B & C & D & E \\
\hline Number (623) & 7 & 6 & 24 & 171 & 415 & 6 & 6 & 22 & 121 & 468 & 1 & 4 & 6 & 25 & 587 \\
\hline
\end{tabular}

ASIA: American Spinal Injury Association.

Table III: Clinical Research Summary

\begin{tabular}{lc}
\hline Characteristics & Total $(\mathbf{n}=623)$ \\
\hline Involved segment, no. of levels & $2.5 \pm 1.3(1-9)$ \\
\hline Anterior approach, no. (\%) & $171(27.4 \%)$ \\
Combined approach, no. (\%) & $135(21.7 \%)$ \\
Posterior approach, no. (\%) & $317(50.9 \%)$ \\
\hline Major complication (59) & $17(2.7 \%)$ \\
Cerebrospinal fluid leakage, no. (\%) & $10(1.6 \%)$ \\
Poor wound healing, no. (\%) & $10(1.6 \%)$ \\
Wound infection, no. (\%) & $13(2.0 \%)$ \\
Local hematoma, no. (\%) & $9(1.4 \%)$ \\
Others, no. (\%) & $42(6.7 \%)$ \\
\hline Recurrence, no. (\%) & $87.8 \%$ \\
\hline Pain-free at the last follow-up, (\%) & \\
\hline
\end{tabular}

All patients were followed up for an average of $34.12 \pm 14.05$ months (range 12-60 months) (Table I). According to the ASIA classification, 208 cases (33.3\%) with neurological deficits had different degrees of postoperative recovery (Table II). The postoperative VAS score was significantly less than the preoperative score $(p<0.05)$, and the VAS score was further significantly reduced at the final follow-up as compared to the postoperative VAS score $(p<0.05)$. The average preoperative ESR was $52.65 \pm 31.29 \mathrm{~mm} / \mathrm{h}$; aside from 42 patients with recurrence, the ESR returned to normal by 3 months after surgery, and no obvious abnormalities occurred at the final follow-up (Figure 2). The fusion rate was $30.2 \%$ and $98.4 \%$ at the 9- and 12-month follow-ups after surgery, respectively. The average fusion time was $11.15 \pm 1.49$ months. Followup radiographs showed all patients had achieved satisfactory fusion (Figure 3A-P).

There were 42 recurrences of spinal tuberculosis. Among these, nine cases were drug-resistant. All the patients with recurrences were cured after reoperation or local drainage, irrigation, and application of antibacterial drugs; no further recurrences had occurred at the final follow-up (Figure 4A-P and $5 \mathrm{~A}-\mathrm{N}$ ).

Fourteen patients experienced graft subsidence or slight movement, but this did not cause physical curve variation or clinical symptoms. Thus, these patients were closely observed, but required no treatments. A total of 59 patients $(9.4 \%)$ suffered from routine surgical complications, including poor postoperative wound healing (including five diabetics), wound infection, local haematoma, cerebrospinal fluid leak, pleural effusion, and postoperative dysphagia (Table III). All were relieved after appropriate treatment.

Details of the clinical outcomes of spinal tuberculosis treated by anterior, posterior and combined approaches are given in Table IV. There was no significant difference in the operative time between the anterior and posterior groups ( $p>0.05)$. The operative time of the combined group was $419.9 \pm 83.6 \mathrm{~min}$, which was longer than the other two groups, and this result had significant differences with the other two groups, respectively 


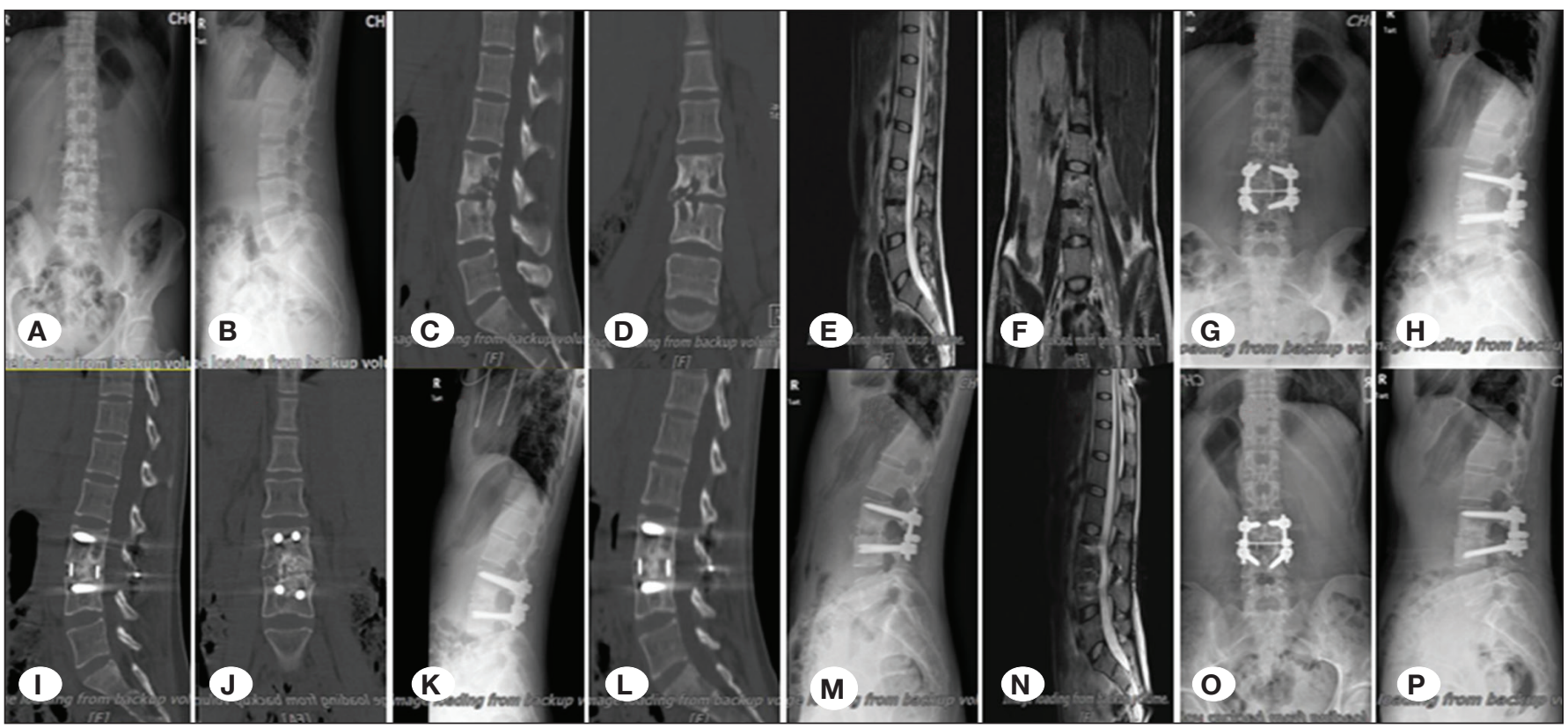

Figure 3: The graph shows a tuberculosis case using a polyether-ether-ketone (PEEK) cage with allograft. Illustration: 24-year-old woman with lumbar spinal tuberculosis. (A, B) preoperative anteroposterior and lateral X-ray films; (C, D) preoperative computed tomography (CT); (E, F) preoperative magnetic resonance imaging (MRI); (G, H) X-ray 9 months after operation; (I, J) CT 9 months after operation; (K) X-ray 15 months after operation; (L) CT 15 months after operation; (M) X-ray 36 months after operation; (N) MRI 36 months after operation; and (O, P) X-ray 48 months after operation.

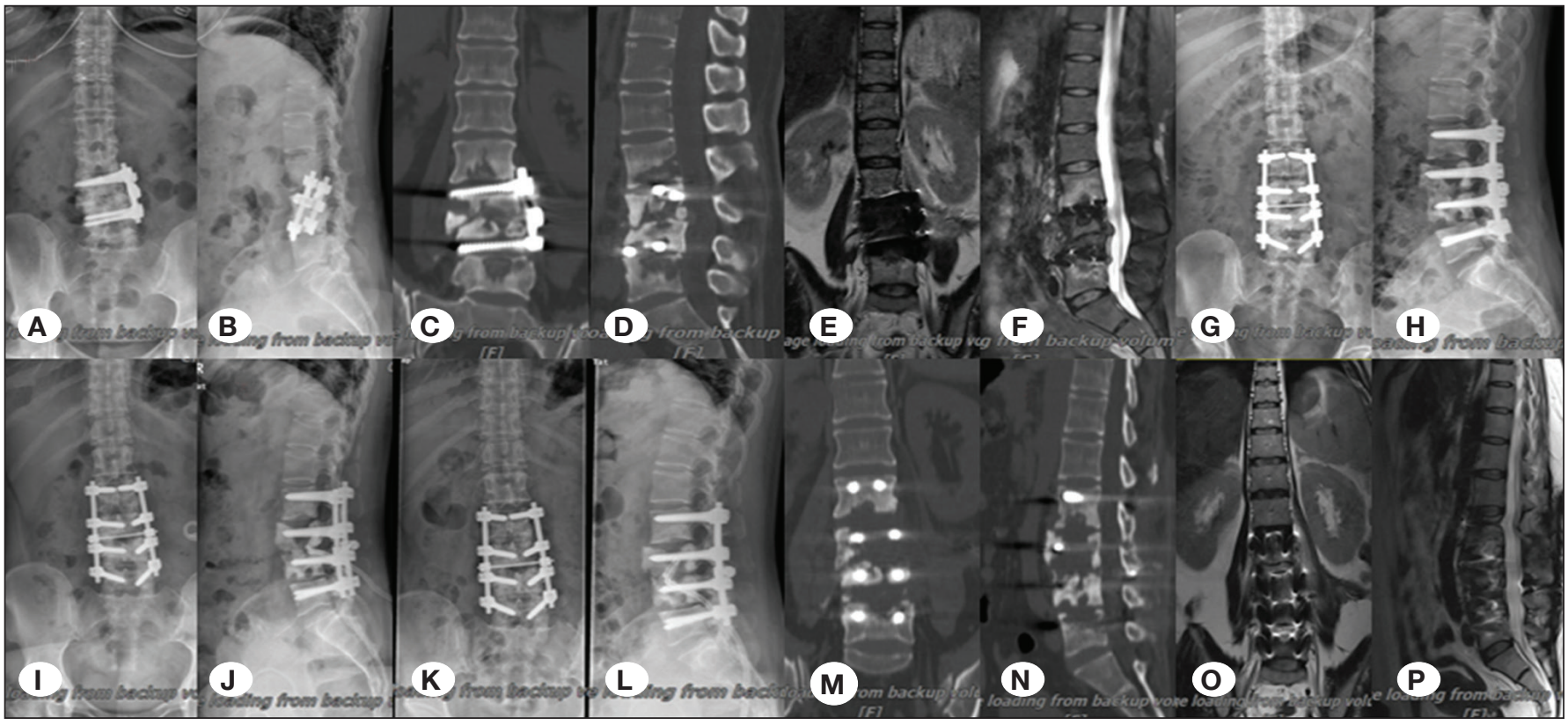

Figure 4: The graph shows a spinal tuberculosis patient requiring revision surgery. Illustration: 37-year-old woman with lumbar spinal tuberculosis (multiple drug resistant tuberculosis [MDR-TB]). (A, B) 72 months after operation at the local hospital, anteroposterior and lateral X-ray films, (C, D) computed tomography (CT) and (E, F) magnetic resonance imaging (MRI); (G, H) immediate postoperative period X-ray films; (I, J) X-ray 3 months after operation; (K, L) X-ray 6 months after operation; (M, N) CT 6 months after operation; and $(\mathrm{O}, \mathrm{P}) \mathrm{MRI} 6$ months after operation. 
$(p<0.05)$. The operative blood loss was as follows: anterior group $619.6 \pm 395.7 \mathrm{~mL}$, posterior group $732.8 \pm 597.1 \mathrm{~mL}$ and combined group $1106.8 \pm 468.2 \mathrm{~mL}$, and there were significant differences among the three groups, respectively $(p<0.05)$. There were also significant differences in the hospitalization time (days) among the anterior, posterior and combined groups $(p<0.05$, respectively). The average time (months) to bone fusion was comparable between the anterior and posterior groups ( $11.1 \pm 2.5$ months vs. $10.6 \pm 2.0$ months, $p>0.05$ ), while the combined group had a longer fusion time as compared with the posterior group (11.6 \pm 2.3 months vs. $10.6 \pm 2.0$ months, $\mathrm{p}<0.05)$. The average postoperative VAS score and ESR at 3 months after the operation was significantly reduced as compared to the preoperative values in the three groups $(p<0.05)$. In addition, the VAS score and ESR was further significantly reduced at the final follow-up as compared to the postoperative VAS score $(p<0.05)$.

\section{DISCUSSION}

The purpose of treatment of spinal tuberculosis is to eliminate the infectious lesions, relieve spinal cord compression, correct spinal deformity and reconstruct spinal stability, and restore normal activities and life. Surgical indications include severe vertebral destruction and kyphosis, spinal cord and nerve dysfunction, dead bone, intractable pain, failed non-operative treatment, and the need to establish a bacteriological diagnosis $(8,28)$. The selection of the surgical treatment approach should be according to the location and extent of the lesion. Individualized treatment and debridement are the key to a successful operation (7).
Debridement inevitably leads to greater bone defects and a further decline in spine stability. The importance of spinal stability reconstruction in the treatment of spinal tuberculosis has been widely accepted. To achieve bone fusion, a fine bone graft and internal fixation is essential; this is one of the principles of the surgical treatment of spinal tuberculosis. However, the best kind of structural bone graft material for specific types of lesions has not yet been determined $(11,36,43)$. Therefore, in this multicentre study, we observed the clinical efficacy of allografts for the treatment of spinal tuberculosis.

The autologous tri-cortical graft harvested from the iliac crest as fusion material is widely used at present. Although it has good clinical efficacy and a high fusion rate, the donor area may suffer from various complications, which brings additional trauma and pain to the patient, prolongs the operative time, and increases blood loss (37). The source for an allograft is relatively abundant and avoids the complications associated with an autograft; bone conductivity is strong and fixation is reliable. It is suitable for large lesions, osteoporosis, and bone deficiency in children. However, there may be a risk of bone resorption and immune rejection. In recent years, with the progress in allograft immunogenicity treatment, allografts are used more frequently in clinics, with satisfactory efficacy. Ozdemir et al. (27) used an allogeneic fibula ring to treat spinal tuberculosis; the fusion rate was $96 \%$. Schuster et al. (33) treated 47 patients with spinal infection using an allograft with combined fixation; they did not observe bone graft non-union or related complications. In a comparative study of allografts and autografts for the treatment of cervical tuberculosis, Bao et al. (2) found that there was no significant difference in the

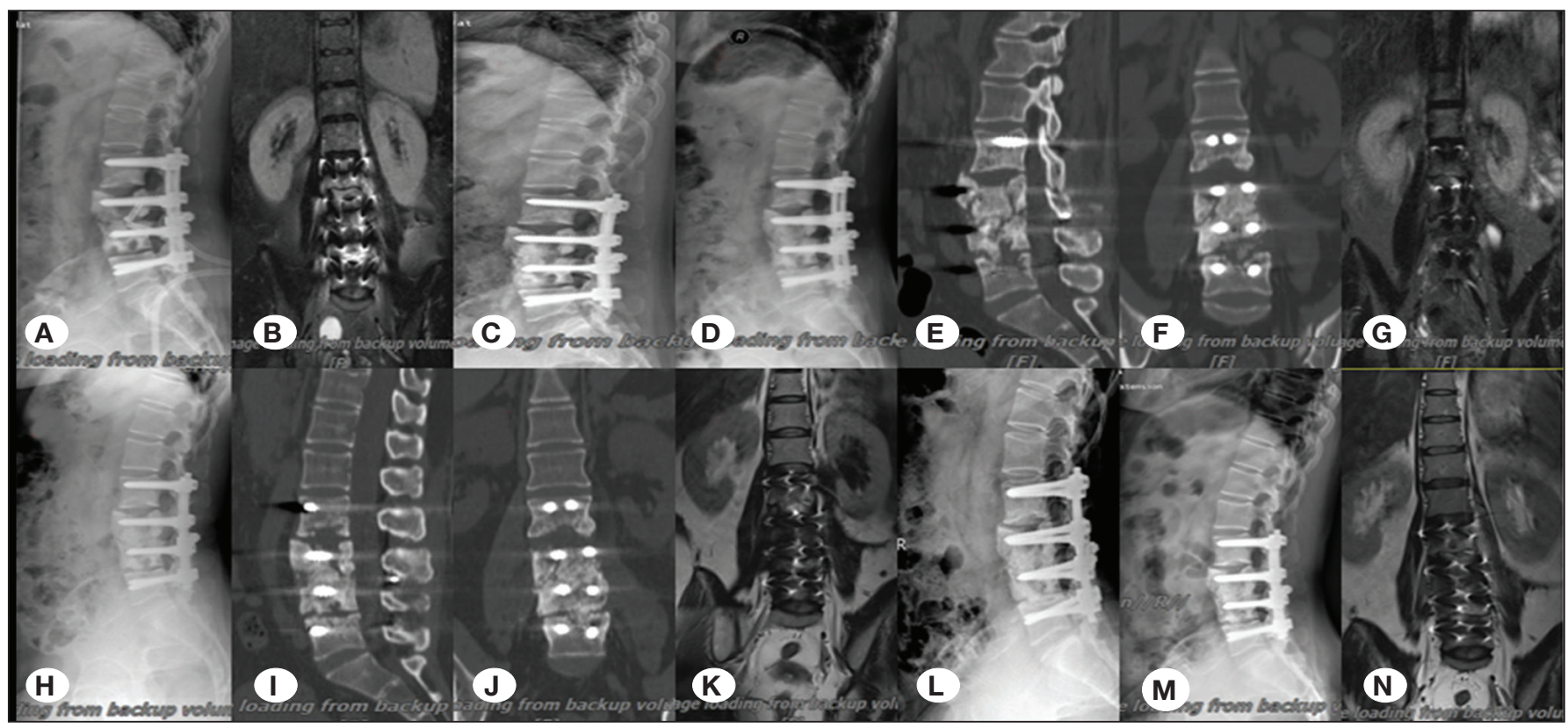

Figure 5: The graph continues to show the above patient (revision). Illustration: (A, B) Eight months after revision for recurrence (preoperative lateral X-ray and MRI); (C) immediate postoperative period X-ray films-revision once again; (D) X-ray 6 months after operation; (E, F) CT 6 months after operation; (G) MRI 6 months after operation; (H) X-ray 12 months after operation; (I, J) CT 12 months after operation; (K) MRI 12 months after operation; (L) X-ray 24 months after operation; (M) X-ray 30 months after operation; and (N) MRI 30 months after operation. 
Yang S. et al: Allograft in Spinal Tuberculosis

Table IV: Clinical Outcomes of Spinal Tuberculosis Treated by Anterior, Posterior and Combined Approach (mean \pm SD)

\begin{tabular}{|c|c|c|c|}
\hline Index & Anterior group & Posterior group & Combined group \\
\hline Operation time (min) & $266.5 \pm 90.7$ & $267.3 \pm 90.3$ & $419.9 \pm 83.6^{\dagger \dagger}$ \\
\hline Blood loss(ml) & $619.6 \pm 395.7$ & $732.8 \pm 597.1^{\dagger}$ & $1106.8 \pm 468.2^{\dagger \dagger \ddagger}$ \\
\hline Hospitalization time (days) & $24.4 \pm 7.7$ & $26.5 \pm 8.9^{\dagger}$ & $30.7 \pm 10.0^{\dagger \dagger \ddagger}$ \\
\hline Follow-up time (months) & $34.1 \pm 6.7$ & $34.2 \pm 6.0$ & $33.7 \pm 7.1$ \\
\hline \multicolumn{4}{|l|}{ ESR } \\
\hline Pre-operation (mm/h) & $53.8 \pm 28.5$ & $52.1 \pm 29.6$ & $51.3 \pm 29.8$ \\
\hline 3 months postoperative $(\mathrm{mm} / \mathrm{h})$ & $9.3 \pm 10.7^{*}$ & $9.3 \pm 6.2^{*}$ & $8.1 \pm 8.3^{\star}$ \\
\hline Pre-operation & $4.7 \pm 1.8$ & $4.7 \pm 1.9$ & $5.0 \pm 1.9$ \\
\hline Post-operation & $2.5 \pm 0.8^{*}$ & $2.5 \pm 1.0^{*}$ & $2.6 \pm 1.0^{*}$ \\
\hline Final follow-up & $0.5 \pm 0.6^{\Delta}$ & $0.5 \pm 0.6^{\Delta}$ & $0.5 \pm 0.6^{\Delta}$ \\
\hline
\end{tabular}

ESR: Erythrocyte Sedimentation Rate, VAS: Visual Analogue Scale

${ }^{\dagger} P<0.05$. Comparison between group Anterior group and Posterior group

${ }^{t+} P<0.05$. Comparison between group Anterior group and Combined group

$\neq P<0.05$. Comparison between group Posterior group and Combined group

${ }^{*} P<0.05$ Comparison between Pre-operation and 3 months postoperative/ Post-operation

${ }^{\Delta} P<0.05$ Comparison between Final follow-up and 3 months postoperative/ Post-operation.

fusion rate between autografts and allografts during followup, and both obtained bone fusion with no complications. In our study of 1196 patients with spinal infection, a total of 623 patients received an allograft. The healing time was 9-18 months (average $11.05 \pm 1.59$ months). This is faster than the healing time reported by Govender $(11,12)$. Although the fusion time was longer than the autograft bone fusion times reported in the literature $(20,22)$, the fusion rate was satisfactory and no complications occurred at the final follow-up.

The use of the cage device has developed rapidly in recent years. It has satisfactory supporting strength and can avoid the need for an autograft or reduce the number of grafts needed; it is becoming a commonly used fusion material in spinal fusion. The literature reports that the titanium cage is not affected by degrading enzymes in an infectious environment, it is resistant to the formation of biofilm on its surface, and it does not increase the risk of infection. These findings provide a strong theoretical basis for the safety of the titanium cage and implant in patients with spinal infection after debridement $(7,14)$. Kuklo et al. (19) reported 21 cases with pyogenic vertebral osteomyelitis treated by combined anterior and posterior approach surgery, with a titanium cage placed through the anterior approach followed by posterior internal fixation. Pain was significantly reduced in 16 patients after the operation. Final follow-up radiographs showed that bone fusion was achieved in all patients. Lu et al. (23) reported 36 cases with pyogenic vertebral osteomyelitis cured by anterior (5 cases), posterior (4 cases), and combined anterior and posterior (27 cases) approaches with an expandable titanium cage. They found there were no implant failures, although two patients had a recurrent infection. Neurological deficits were improved in all cases, and pain disappeared in $81 \%$ of patients. Up to now, in the area of spinal infectious disease, there have been few studies on PEEK material. According to the successful experience with the titanium cage in spinal infection, some patients (492 cases) in this study attempted to use the PEEK cage for bone grafting. The result showed all patients achieved bone fusion within a year. There was significant relief of pain and maintenance of spinal curvature in all patients, and no patient suffered material-related infection.

The surgical approaches for spinal tuberculosis include anterior, posterior, and combined anterior and posterior approaches $(7,17,27)$. The anterior approach allows for debridement and decompression to be performed better under direct vision, regain of intervertebral height, and successful bone graft fusion. We found that the anterior approach group had less blood loss and hospitalization time; these results are consistent with previous studies $(6,20)$. However, bone graft collapse can also occur, as well as correction loss and kyphosis exacerbation. Combined surgery is able to achieve better spinal stability after removal of multiple vertebrae and reduce fusion range. In addition, posterior pedicle screw 
internal fixation is in accordance with the biomechanical characteristics of the spine and enhances the stability of the posterior column. At the same time, debridement and internal fixation were separated to reduce the spread of pathogens (3). However, the combined approach could result in more serious trauma and higher complication rates (16). In the present study, 135 patients with spinal tuberculosis received combined anterior and posterior procedures, which led to an increase in operating time, more blood loss and prolonged hospitalization time. As a result, it is particularly not suitable for elderly patients in poor health. The posterior approach is relatively simple, allows complete debridement and decompression and reconstructs spinal stability in a single incision. At the same time, it also effectively corrects kyphosis and reduces internal fixation-related complications (34). However, the posterior approach also has some disadvantages, including insufficient visibility, longer learning curve, dural tears, and adhesion (41). It also destroys the posterior column and affects stability (42). Our results suggest that posterior surgical treatment of spinal tuberculosis is similar to anterior debridement and bone grafting in terms of therapeutic efficacy, which is consistent with previous research (38). For the treatment of spinal infectious diseases, anterior, posterior or combined anterior and posterior approaches can all achieve satisfactory therapeutic effects (5). According to our experience, we suggest that the appropriate surgical procedure should be selected according to the properties of the infection type, pathological results, lesion range, and general physical condition of the patient. As long as the indications are appropriate, satisfactory efficacy can be achieved, no matter what kind of surgical approach is taken $(15,30,44)$.

In terms of complications, 14 patients (1.9\%) suffered bone graft-related complications; however, there were no obvious differences compared with previous reports, and the incidence of complications was low $(9,24)$. In addition, the complication rates associated with the surgeries were comparable with previously published rates $(7,22,26)$. There are few reports comparing the recurrence rates between allografts and autografts in spinal fusion for spinal tuberculosis. Mikhael et al. (25) retrospectively reviewed 1435 patients with spinal infection who underwent spinal fusion surgery, including 144 patients with an irradiated allograft, 441 patients with a non-irradiated allograft, and 850 patients with an autograft. They found that local recurrence rates were $1.7 \%, 3.2 \%$, and $4.3 \%$ respectively, with no significant differences $(p=0.51)$. In our study, there were $42(6.7 \%)$ cases of recurrence, which were primarily among drug-resistant tuberculosis patients $(9 / 36)$. This is associated with the time-consuming nature of traditional drug susceptibility testing and delayed diagnosis of resistance. From the beginning of 2012, two hospitals specializing in this research began to utilize molecular techniques to diagnose drug-resistant tuberculosis. The detection time has been shortened to 6-12 hours, and there has been a greatly improved detection rate for drug resistant tuberculosis, which allows for the timely adjustment of chemotherapeutic regimens, and remarkably reduces the risk of recurrence of spinal tuberculosis.
Although this study has achieved satisfactory results, there are still some limitations. Firstly, this study represents retrospective research, and the short follow-up time may affect the reliability of the evaluation results (39). Secondly, different hospitals have different surgical proficiency and capability, which may cause a certain degree of bias in the findings.

\section{CONCLUSION}

Allografts can achieve satisfactory clinical results when applied in the surgical treatment of spinal tuberculosis. Although the fusion time of allografts was a little longer than that of autografts, solid fusion was achieved by 9-18 months.

\section{REFERENCES}

1. Banwart JC, Asher MA, Hassanein RS: Iliac crest bone graft harvest donor site morbidity. A statistical evaluation. Spine (Phila Pa 1976) 20:1055-1060, 1995

2. Bao D, Ma YZ, Chen X, Li HW, Guo LX, Gao TJ: Exploratory development of titanium mesh with bone allograft in treatment of cervical spinal tuberculosis. Zhonghua Wai Ke Za Zhi 48: 112-115, 2010

3. Chen WJ, Wu CC, Jung $\mathrm{CH}$, Chen LH, Niu CC, Lai PL: Combined anterior and posterior surgeries in the treatment of spinal tuberculous spondylitis. Clin Orthop Relat Res 398:5059, 2002

4. Chhabra N, Gupta N, Aseri ML, Mathur SK, Dixit R: Analysis of thyroid function tests in patients of multidrug resistance tuberculosis undergoing treatment. J Pharmacol Pharmacother 2:282-285, 2011

5. Cui X, Li LT, Ma YZ: Anterior and posterior instrumentation with different debridement and grafting procedures for multilevel contiguous thoracic spinal tuberculosis. Orthopaedic Surgery 8:454-461, 2016

6. Dai LY, Jiang LS, Wang W, Cui YM: Single-stage anterior autogenous bone grafting and instrumentation in the surgical management of spinal tuberculosis. Spine (Phila Pa 1976) 30: 2342-2349, 2005

7. Dimar JR, Carreon LY, Glassman SD, Campbell MJ, Hartman MJ, Johnson JR: Treatment of pyogenic vertebral osteomyelitis with anterior debridement and fusion followed by delayed posterior spinal fusion. Spine (Phila Pa 1976) 29: 326-332; discussion 332, 2004

8. Emery SE, Chan DP, Woodward HR: Treatment of hematogenous pyogenic vertebral osteomyelitis with anterior debridement and primary bone grafting. Spine (Phila Pa 1976) 14:284-291, 1989

9. Faraj AA, Webb JK: Spinal instrumentation for primary pyogenic infection report of 31 patients. Acta Orthop Belg 66: 242-247, 2000

10. Fayazi AH, Ludwig SC, Dabbah M, Bryan Butler R, Gelb DE: Preliminary results of staged anterior debridement and reconstruction using titanium mesh cages in the treatment of thoracolumbar vertebral osteomyelitis. Spine J 4:388-395, 2004

11. Govender S: The outcome of allografts and anterior instrumentation in spinal tuberculosis. Clin Orthop Relat Res 398:60-66, 2002 
Yang S. et al: Allograft in Spinal Tuberculosis

12. Govender S, Kumar KP: Cortical allografts in spinal tuberculosis. Int Orthop 27:244-248, 2003

13. Grogan DP, Kalen V, Ross TI, Guidera KJ, Pugh LI: Use of allograft bone for posterior spinal fusion in idiopathic scoliosis. Clin Orthop Relat Res 369:273-278, 1999

14. Ha KY, Chung YG, Ryoo SJ: Adherence and biofilm formation of Staphylococcus epidermidis and Mycobacterium tuberculosis on various spinal implants. Spine (Phila Pa 1976) 30:38-43, 2005

15. He Q, Xu J: Comparison between the antero-posterior and anterior approaches for treating L5-S1 vertebral tuberculosis. Int Orthop 36: 345-351, 2012

16. Hee HT, Majd ME, Holt RT, Pienkowski D: Better treatment of vertebral osteomyelitis using posterior stabilization and titanium mesh cages. J Spinal Disord Tech 15:149-156; discussion 156, 2002

17. Jin WD, Wang Q, Wang ZL, Geng GQ: Complete debridement for treatment of thoracolumbar spinal tuberculosis: A clinical curative effect observation. Spine Journal 14:964-970, 2014

18. Kourbeti IS, Tsiodras S, Boumpas DT: Spinal infections: Evolving concepts. Curr Opin Rheumatol 20:471-479, 2008

19. Kuklo TR, Potter BK, Bell RS, Moquin RR, Rosner MK: Singlestage treatment of pyogenic spinal infection with titanium mesh cages. J Spinal Disord Tech 19:376-382, 2006

20. Lan J, Tang X, Xu Y, Wang S, Zhang Z, Zheng J, Wang F, Chen Y, Cai Q, Ran M, Liu Y: Anterior approach debridement and bone grafting with self-locked titanium plate internal fixation for treating lumbosacral tuberculosis. Zhongguo Xiu Fu Chong Jian Wai Ke Za Zhi 30:585-589, 2016

21. Lee CK, Vessa P, Lee JK: Chronic disabling low back pain syndrome caused by internal disc derangements. The results of disc excision and posterior lumbar interbody fusion. Spine (Phila Pa 1976) 20:356-361, 1995

22. Li L, Xu J, Ma Y, Tang D, Chen Y, Luo F, Li D, Hou T, Zhou $Q$, Dai $F, H e Q$, Zhang Z: Surgical strategy and management outcomes for adjacent multisegmental spinal tuberculosis: A retrospective study of forty-eight patients. Spine (Phila $\mathrm{Pa}$ 1976) 39:E40-48, 2014

23. Lu DC, Wang V, Chou D: The use of allograft or autograft and expandable titanium cages for the treatment of vertebral osteomyelitis. Neurosurgery 64:122-129; discussion 129-130, 2009

24. Matsui H, Hirano N, Sakaguchi Y: Vertebral osteomyelitis: An analysis of 38 surgically treated cases. Eur Spine J 7:50-54, 1998

25. Mikhael MM, Huddleston PM, Nassr A: Postoperative culture positive surgical site infections after the use of irradiated allograft, nonirradiated allograft, or autograft for spinal fusion. Spine (Phila Pa 1976) 34:2466-2468, 2009

26. Ogden AT, Kaiser MG: Single-stage debridement and instrumentation for pyogenic spinal infections. Neurosurg Focus 17:E5, 2004

27. Ozdemir HM, Us AK, Ogun T: The role of anterior spinal instrumentation and allograft fibula for the treatment of pott disease. Spine (Phila Pa 1976) 28:474-479, 2003
28. Park KH, Cho OH, Lee YM, Moon C, Park SY, Moon SM, Lee JH, Park JS, Ryu KN, Kim SH, Lee SO, Choi SH, Lee MS, Kim YS, Woo JH, Bae IG: Therapeutic outcomes of hematogenous vertebral osteomyelitis with instrumented surgery. Clin Infect Dis 60:1330-1338, 2015

29. Pola E, Rossi B, Nasto LA, Colangelo D, Logroscino CA: Surgical treatment of tuberculous spondylodiscitis. Eur Rev Med Pharmacol Sci 16 Suppl 2:79-85, 2012

30. Pu X, Zhou Q, He Q, Dai F, Xu J, Zhang Z, Branko K: A posterior versus anterior surgical approach in combination with debridement, interbody autografting and instrumentation for thoracic and lumbar tuberculosis. Int Orthop 36:307-313, 2012

31. Revell CM, Athanasiou KA: Success rates and immunologic responses of autogenic, allogenic, and xenogenic treatments to repair articular cartilage defects. Tissue Eng Part B Rev 15:1-15, 2009

32. Safran O, Rand N, Kaplan L, Sagiv S, Floman Y: Sequential or simultaneous, same-day anterior decompression and posterior stabilization in the management of vertebral osteomyelitis of the lumbar spine. Spine (Phila Pa 1976) 23:1885-1890, 1998

33. Schuster JM, Avellino AM, Mann FA, Girouard AA, Grady MS, Newell DW, Winn HR, Chapman JR, Mirza SK: Use of structural allografts in spinal osteomyelitis: A review of 47 cases. J Neurosurg 93:8-14, 2000

34. Senel A, Kaya AH, Kuruoglu E, Celik F: Circumferential stabilization with ghost screwing after posterior resection of spinal metastases via transpedicular route. Neurosurg Rev 30:131-137; discussion 137, 2007

35. Shi J, Wang Z, Wang P, Jin W: Application and comparison of allograft and autograft bone for interbody fusion in cervical tuberculosis. Zhongguo Xiu Fu Chong Jian Wai Ke Za Zhi 25:1290-1293, 2011

36. van Vugt TA, Geurts J, Arts JJ: Clinical application of antimicrobial bone graft substitute in osteomyelitis treatment: A systematic review of different bone graft substitutes available in clinical treatment of osteomyelitis. Biomed Res Int 2016:6984656, 2016

37. Wang B, Lv G, Liu W, Cheng I: Anterior radical debridement and reconstruction using titanium mesh cage for the surgical treatment of thoracic and thoracolumbar spinal tuberculosis: Minimium five-year follow-up. Turk Neurosurg 21:575-581, 2011

38. Wang $Y X$, Zhang $H Q$, Li $M$, Tang MX, Guo CF, Deng A, Gao Q, Wu JH, Liu JY: Debridement, interbody graft using titanium mesh cages, posterior instrumentation and fusion in the surgical treatment of multilevel noncontiguous spinal tuberculosis in elderly patients via a posterior-only. Injury 48:378-383, 2017

39. Wu WJ, Lyu JT, Liu X, Luo F, Hou TY, Zhou Q, Li Z, Chen Y, Li LT, Zheng Y, Wang G, Xu JZ, Zhang ZH: Surgical treatment of thoracic spinal tuberculosis: A multicenter retrospective study. World Neurosurgery 110:e842-e850, 2018

40. Zavattero E, Boffano P, Bianchi FA, Bosco GF, Berrone S: The use of titanium mesh for the reconstruction of defects of the anterior wall of the frontal sinus. J Craniofac Surg 24:690-691, 2013 
41. Zeng Y, Chen ZQ, Guo ZQ, Qi Q, Li WS, Sun CG: The posterior surgical treatment of old tuberculous kyphosis. Zhonghua Wai Ke Za Zhi 50:23-27, 2012

42. Zhang H, Sheng B, Tang M, Guo C, Liu S, Huang S, Gao Q, Liu J, Wu J: One-stage surgical treatment for upper thoracic spinal tuberculosis by internal fixation, debridement, and combined interbody and posterior fusion via posterior-only approach. Eur Spine J 22:616-623, 2013
43. Zhang X, Zhang Z, Zhang Y, Wang J, Lu M, Hu W, Wang Y, Ma $X$, Wang $Y$ : Minimally invasive retroperitoneoscopic surgery for psoas abscess with thoracolumbar tuberculosis. Surg Endosc 29: 2451-2455, 2015

44. Zhang ZX, Li T, Hao DJ: Single-stage treatment of osteomyelitis of the cervical spine using anterior instrumentation and titanium mesh cages. Spine (Phila Pa 1976) 41:E949-954, 2016 